\title{
LECTURE
}

\section{The Diagnosis of Nonanginal Chest Pain}

\author{
Jules Constant \\ Department of Internal Medicine, State University of New York at Buffalo, NY, USA
}

(Received for publication on March 27, 1990)

\begin{abstract}
The term "atypical chest pain" is a waste-basket term that leads physicians to send any patient with chest pain to coronary angiography. In order to avoid this term, we must learn to distinguish atypical angina from nonanginal chest pain before angiography is considered in order to avoid unnecessary invasive procedures. A chest pain is very likely nonanginal if its duration is over 30 minutes or less than 5 seconds, it increases with inspiration, can be brought on with one movement of the trunk or arm, can be brought on by local fingers pressure, or bending forward, or it can be relieved immediately on lying down. There are also many presumptive signs of nonanginal chest pain such as localization with one finger, radiation to the nuchal area, an inframammary primary site, a pain that reaches maximum at the onset, or relief within a few seconds of swallowing food. Cervical root compression pain and esophageal spasm are the greatest mimics of angina since they can both be relieved by nitroglycerin but they have several features which help to rule out angina. (Keio J Med 39 (3): 187-192, September 1990)
\end{abstract}

Key words: angina, costochondritis, cervical root compression syndrome, esophageal dysfunction, hyperventilation syndrome

\section{Introduction}

There is an urgent need for a new look at chest pain with a view to trying to distinguish atypical angina from nonanginal chest pain. Even when physicians diagnose the chest pain as classic or typical angina, in some centers as high as 30 percent of such patients had normal coronary arteries on angiography. ${ }^{1-3}$ It should, therefore, not be surprising to find that when the pain was said to be atypical angina, as many as 80 percent of patients were found to have normal coronary angiograms. ${ }^{4}$ The disillusionment of physicians who saw too many false positives and negative coronary angiograms in patients with chest pain has caused many physicians to send patients with almost any chest pain directly to angiography. This approach was encouraged by the introduction of a new terminology into the cardiological literature. Physicians were told that all chest pains could be divided into two categories, either classic angina or atypical chest pain, i.e., atypical angina and nonangina were combined into a single term. The term "atypical chest pain" implies that it is impossible to differentiate atypical angina from the nonanginal chest pain without an angiogram. The term "atypical chest pain" is being used to send to angiography anyone with any kind of chest pain.
Since there are no studies that conclusively prove that certain types of chest pains are non-anginal, it would appear unscientific to label a chest pain as nonanginal simply by force of reason and our knowledge of physiology, as well as our experience. But for some types of chest pains rigidly controlled scientific proofs may not be necessary. For example, no one would call angina a recurrent chest pain that on many occasions lasts two or three days without cease. Yet patients have been sent to angiography for just such chest pains because most of the time their pains last 10 or 15 minutes, a perfect duration for classic angina. This may be because when a physician asks the patient how long the pain lasts, the patient will often not volunteer maximum or minimum duration but gives the average duration.

Asking the patient questions to primarily diagnose the pain as nonanginal is such an unfamiliar concept that physicians must be warned of the many traps into which they may fall. For example, it is easily forgotten that the patient may have more than one pain, only one of which may be anginal, yet the patient may not separate them when answering your questions. It has been estimated that 50 percent of patients with angina also have musculoskeletal chest pain. ${ }^{5}$ In other studies up to one-half of patients with chest pains due to costochondritis had 
angina as well. ${ }^{6-8}$

The first admonition, then, is to be sure that the pain you are inquiring about is the same one that the patient is describing so that separate questions are asked concerning each pain. Other less obvious traps into which the unwary physician may fall will be discussed separately with each nonanginal symptom.

\section{Maximum Duration Criteria}

In dogs myocardial necrosis occurs after only about one-half hour of coronary ligation. From the results of exercising men with classic angina and known coronary disease, it has been found that angina rarely lasts more than 20 minutes. ${ }^{9}$ Since a patient does not use a stopwatch to time his angina and there may be some exceptions, we should probably use 30 minutes as the maximum duration of pain. Emotion-induced angina or vasospastic angina also lasts no longer that 30 minutes. Indeed the literature actually suggests that the pain of variant or vasospastic angina tends to be shorter than the usual type of angina. ${ }^{10}$

In determining the duration of a patient's chest pain there are several traps of which it is well to be aware.

1. If the pain lasts over 30 minutes, it is essential to ask whether the pain is accompanied by palpitations. A tachycardia may be causing the angina and the pain will last as long as the tachycardia.

2. If the history reveals that only one of the patient's recurrent episodes of chest pain lasted over 30 minutes, it may have been due to infarction. Therefore it is necessary to ask if at least two or three such long-lasting episodes occurred before deciding that the pain is non-anginal.

3. If the patient lies down with the pain, angina may persist for over the 30 -minute period because of the increased venous return and blood volume that occurs in the supine position. Most patients with angina never lie down with their pain; if they do, they learn quickly that lying down is not the best position for angina. The occasional patient, however, does not seem to realize that all ill feelings are not best managed by lying down.

4. If the patient claims that the pain lasts more than 30 minutes, it may mean that the patient really had multiple short episodes, each one being too short for angina (i.e., lasting only a few seconds.)

\section{Minimum Duration Criteria}

If a blood pressure cuff on the arm is held above systolic pressure for a few minutes, only some numbness and tingling are felt in the hand. If, however, the hand is exercised for a few minutes, severe pain is produced. This pain is due to a chemical produced by the muscle exercising with inadequate oxygen supply. When it occurs in the legs, it is called claudication. Therefore angina may be considered to be cardiac muscle claudication and is presumably also produced by a chemical mediator, probably one of the kinins. Since it takes time for a chemical to reach maximum effect, and then subside, angina should be crescendo-decrescendo in nature and cannot be a short stab. ${ }^{11}$ Although a lightning stab that disappears immediately has long been recognized by cardiologists as nonanginal, little thought has been given to how brief the pain of angina can be if it is longer than just a stab. Five seconds is a new concept that has been found to be a reliable cutoff point in distinguishing angina from non-angina. ${ }^{11}$ It is a common experience that if chest pains are of less than 5 seconds duration, the patient will have either a normal coronary angiogram or have other nonanginal characteristics to the pain. Chest pains that last only a few seconds should be considered nonanginal with the following exceptions:

1. If the patient with exercise-induced angina is so aware of the first signs of discomfort that he stops his activity the moment he feels the onset of pain, then his pain may disappear within a few seconds. Therefore you must ask for the exact circumstances under which the pain lasted for less than 5 seconds. This implies, of course, that if the pain occurs at rest and lasts less than 5 seconds, it is nonanginal.

'2. If you ask patients whether the pain lasts for just a few seconds, they will often say yes, even if it actually lasts longer. Tap out four beats at 1 -second intervals and ask if the pain can disappear with the last tap. When the time interval is made this precise, patients will commonly tell you that the pain always lasts longer than that and they really mean 15 to 30 seconds. The patient may assume that the exact duration of the pain is not important and that the physician does not require such precise information. Patients often assume that up to 30 seconds is a reasonable duration to report as a "few seconds."

The second reason for giving a wrong answer is that they are ignoring the 10 to 30 seconds of discomfort that occurred before stopping the exertion or taking a nitrite, i.e., they really mean that the pain lasts less than 5 seconds after stopping the exercise or taking a nitrite even though it has been present for perhaps as long as 30 seconds before stopping the exertion.

3. The patient may state that the pain lasts for a few minutes but actually means that there are short stabs of pain, each lasting less than 5 seconds, occurring repeatedly over a few minutes.

\section{Respiratory Movement Criteria}

Since it is unlikely that cardiac claudication can be 
induced by one deep inspiration, a chest pain that increases with one inspiration should be considered nonanginal. Although there has actually been a report of a patient who was said to have had "probable angina" whose pain increased with inspiration, this patient had no coronary angiography, and it is no surprise that his stress test was negative. ${ }^{12}$

One pitfall concerning the effect of respiration is the misinterpretation by the patient of your question to mean repeated inspiration. Hyperventilation can conceivably make angina worse by increasing venous return to the heart, as well as by causing coronary vasoconstriction. ${ }^{13}$ The question, therefore, must not be whether the pain increases with deep breathing but whether one deep breath can either bring on the pain or make it worse.

\section{Arm and Trunk Movement Criteria}

Although it should be obvious that angina ought not to be brought on by a single movement of the arm or trunk, this is one of the commonest nonanginal symptoms missed by the physician. The difficulty here is that patients with true angina may say that arm movements can bring on their chest pain if they interpret the question to mean repeated arm movement, as when cleaning a window. Repeated arm movements have in fact been shown to increase oxygen consumption more than the same amount of work done by the more efficient leg muscles. ${ }^{14}$

\section{Chest Tenderness Criteria}

Cardiac claudication cannot be produced by local finger pressure applied to the chest wall. Therefore the site of anginal pain should not be tender. This seems to contradict some literature describing "angina" appearing after myocardial infarction that was often reproducible by pressing on trigger areas. ${ }^{15}$ These pains following infarction are now recognized as being nonanginal and have been called "causalgia" in the English literature, and "chondrodynia" in French reports. ${ }^{16,17}$ Fortunately, they can often be eliminated permanently by one or more treatments with a cold spray or local injection of lidocaine. ${ }^{15}$

The occasional patient, however, will have a tender area at the site of chest pain if he experiences anginal pain that is referred to an area of previous trauma. Some bizarre sites of referral of anginal pain have been attributed to the phenomenon of facilitation in which pain impulses reaching the thoracic cord from angina, can follow pathways previously established by a somatic injury. ${ }^{18}$ Under these circumstances anginal pain has been thought to be referrable to such unlikely places as the top of the head.

\section{Body Position Criteria}

If the patient reports that his pain is relieved within a few seconds of lying down, this is nonanginal pain because the immediate increase in venous return on assuming the horizontal position can enlarge the heart and increase its oxygen consumption. It is a common observation that patients with angina rarely ever lie down to get relief. The difficulty here is that almost all patients with angina who lie down will ultimately get relief from their pain because of the resting state and slower heart rate, both of which will gradually overcome the increase in heart size. The question that must be asked is whether the pain improves within a few seconds of lying down.

If the pain is brought on immediately upon stooping forward, it is probably not angina. One of the characteristics of esophageal pain, which one study used to differentiate it from angina, was its precipitation by bending forward. ${ }^{19,20}$ Pain caused by gas in the stomach or splenic flexure may also be brought on by stooping; but the clue here is immediate relief on belching or passing gas. When the gas produces a stabbing pain that extends to the left pectoral region, it has been called the "magenblas syndrome," "splenic flexure syndrome," or "pseudoangina."

The trap here is that angina can often be brought on if the patient is actually doing work with his hands while he is in the bent or stooped position. Even the time it takes to tie shoelaces while bent forward may be long enough to produce true angina. The pain must come on within a few seconds of bending forward without doing any work with the hands in order to be classified as nonanginal.

\section{Relieving Factor Criteria}

If the pain is relieved within a few seconds by one or two swallows of water or food, it is nonanginal pain. Either esophageal spasm or achalasia are the most likely etiologies here, especially if the pain can be precipitated by a cold drink or relieved by a warm one. ${ }^{20,21}$ The problem here is that about 5 percent of patients with true angina will claim that they get relief with antacids. However, only with esophageal spasm will the pain diminish immediately. Here a word of caution is necessary about the patient's interpretation of "immediately" which is often taken to mean a few minutes.

\section{Presumptive Evidence of Nonanginal Chest Pain}

The nonanginal signs presented thus far are all strong enough to make you deny the presence of angina. There are, however, other signs that although they cannot be taken as certain signs of nonangina, they may be considered presumptive evidence. One of these is the presence of pain so sharply localized that the patient points 
to it with one finger. It is well known that a visceral pain is difficult to localize. Therefore, if you ask the patient to show you where the pain is, he will usually use a fist or the entire hand. However, if the question that you ask the patient is to point to the site of the pain, then one finger may very well be used even for true angina. If you simply ask where the pain is, and one finger is used to point to the site, it is more likely to be nonanginal.

If we assume that anginal pains are primarily caused by a chemical mediator, the mode of onset of angina should theoretically always be gradual. Therefore, if the pain comes on with its maximum intensity at its onset, it is strong presumptive evidence for nonanginal chest pain.

The presence of left inframammary pain is also an unproven presumptive site of nonanginal pain. There is a strong tradition, especially in British teaching, that left intramammary pain is nonanginal. ${ }^{22,23}$ It is so common to have other nonanginal signs, such as local tenderness, in patients whose primary site is inframammary, especially in women, that it may well prove to be true that such a site is nonanginal. However, this has yet to be confirmed, and in isolation, i.e., without other nonanginal signs, should only be considered presumptive evidence. The common nonanginal left inframammary pain was thought by Paul Wood to be an intercostal muscle pain caused by either the insistent minimal trauma of an over-active heart, or by fatigued and strained respiratory muscles which by poor control over respiratory movement caused by a functional disturbance, would be overworked. ${ }^{24}$

Another presumptive nonanginal sign concerns the site of radiation. In a series of patients with coronary disease and anginal pain referred to the neck, the pain was always described as going to the anterior neck, i.e., the throat. However, in patients with neck pain but angiographically normal coronary arteries, almost half reported pain confined to the posterior cervical muscle mass. ${ }^{25}$ If the pain begins low in the anterior or posterior chest and goes to the back of the neck, perhaps esophageal and not anginal pains should be the first consideration. One of the difficulties here is that the primary site of true angina may be high in the anterior and posterior chest, and this kind of pain may reach the back of the neck. However, experience has also shown that anginal pain will not go to the nuchal area of the neck, i.e., it will not be felt over the occipital protuberance. If the chest pain radiates to this nuchal area, it should be considered as probably nonanginal. It is usually not enough to simply ask about radiation to the back of the neck; have the patient point to the exact site to see if the site touched is the occiput.

Do not fall into the trap of thinking that you are diagnosing nonanginal chest pain and forget to ask whether the patient has a tightness or pressure which, indeed, may be due to angina. Most patients do not interpret pressure or tightness as pain. It is not uncommon for a patient to have nonanginal chest pains but have typical angina in terms of pressure or tightness.

If your history is inconclusive because of the inability of the patient to answer your questions, it may be necessary to have the patient return after trying the effect of respiration, local pressure, or arm movements, or even timing the pain with a watch before a decision can be made. Only if there are no nonanginal symptoms may we conclude that the patient has either atypical or classic angina.

Of course, finding the cause of the pain is another method of diagnosing the presence of nonanginal chest pain. To find the cause of nonanginal chest pains requires you to be familiar at least with the following entities:

1. "The precordial catch" which is related to a slouched or bent over posture, and usually lasts only seconds. ${ }^{26}$

2. Cervical root compression chest pain ${ }^{27}$ which can be reproduced by having the patient reach around the front of his chest and try to touch the opposite scapula. It is specifically relieved by nitrates. The nerve root passes through a bony canal accompanied by an artery and vein. If the vein becomes engorged, as with the venous return of walking, it may exert pressure on the adjacent artery and nerves. Nitroglycerin causes pooling of venous blood in the legs and will allow decompression of the cervical nerve root. These hemodynamic effects could account for the ability of the cervical compression syndrome to strongly mimic angina.

3. Esophageal retrosternal pain, which can be relieved or exacerbated by swallowing foods or hot liquids. ${ }^{21}$ Nitroglycerin will relieve esophageal pain, but unlike its effect on angina, esophageal pain will usually not be relieved in less than three minutes. ${ }^{20,28}$ Esophageal pain can be precipitated by bending over or by drinking very hot or very cold liquids. ${ }^{20}$ This is strongly suggested if associated with dysphagia, and can be definitively diagnosed by reproduction with acid perfusion into the esophagus, or by noting abnormal esophageal manometry. (S-T changes on intubation in a subject with esophageal abnormalities suggest both ischemic heart disease and esophageal dysfunction.

4. The hyperventilation syndrome. Chest pain here may be due to relatively fixed muscles of the chest and diaphragm causing the muscles of the upper chest to be overstretched. Dizziness, lightheadedness, dyspnea, and paresthesias of the fingers, toes, and circumoral area are common. There is commonly an abortive breath-holding time of less than 20 seconds. ${ }^{30}$

5. Chest wall syndromes. The many causes of nonanginal pains associated with chest wall tenderness may be combined under the name of "chest wall 
syndromes". 6,7

a. Costochondritis can be reproduced by pressure over the costal cartilages. It is Tietze's syndrome only if there is local swelling as well. ${ }^{8}$

b. Thoracic root pains are reproduced by running the fingernail down the back to elicit a hyperalgesic belt.

c. Xiphodynia can be reproduced by pressure on the xiphisternum, may radiate to the chest and arms, and is completely relieved by lidocaine infiltration. ${ }^{31}$

d. Pectoral myofascitis or biceps tendonitis can be reproduced by squeezing the pectoral muscles or the head of the biceps, respectively.

A protocal that may assist the physician in asking the nonanginal questions follows.

1. Where is the pain? Note whether the patient points with one finger.

2. Is there more than one pain? If so, ask about each one separately.

3. Is there a tightness or pressure sensation together with or separate from the pain?

4. Does the discomfort last over 30 minutes? If yes,

a. On how many occasions?

b. With palpitations?

c. After lying down?

d. Repeated stabs over 30 minutes.

5. Does the discomfort last only a few seconds? If yes, tap out 5 seconds. Under what circumstances, i.e., after stops walking, or at rest?

6. Does it increase with one deep inspiration?

7. Can one movement of an arm or of the trunk bring it on?

8. Can local pressure with your fingers make it worse or bring it on?

With continued experience in recognizing nonanginal chest pain, it is possible that even in the presence of significant coronary obstruction or angiography, a physician will have the confidence to deny that the patient's chest pain is ischemic. Where there is no clear concept in the physician's mind of nonanginal chest pain, there are instances where even when an obvious nonanginal symptom was elicited, it was ignored. ${ }^{2} \mathrm{I}$ have seen one angiographer label as angina a pain that lasted as long as 4 or 5 hours every day for months simply because the patient had an abnormal coronary angiogram. Evidence that nonanginal questions are not asked was impressed upon me when I saw a patient with three nonanginal symptoms which were not elicited by two excellent cardiologists who called the pain "classic angina," despite, to their surprise, normal coronary angiograms and no cardiomyopathy.

The tendency on the part of many physicians to overlook asking nonanginal questions should cause us to view with skepticism articles that claim to show normal cor- onary angiograms in the presence of "typical angina." There is no doubt that true angina and normal coronary angiograms occasionally occur, and is presumably accounted for either by spasm or by obstruction of deep intramural arteries (not known as syndrome $\mathrm{X}$ ). But the pain described in these patients should be anginal, i.e., there should be no known nonanginal characteristics to their pain.

A patient with a nonanginal cause of chest pain may have several classic anginal symptoms with his nonanginal pains. Even one reliable nonanginal symptom should cancel any number of symptoms of classic angina. In order not to be misled by the patient's classic anginal symptoms, it is helpful to ask the nonanginal questions first. This may make it easier to ignore any number of symptoms of classic angina if one dependable nonanginal symptom is present. A symptom complex with a nonanginal component should not be called "atypical angina," or atypical chest pain. Atypical angina should be applied to a pain that has one or more unusual characteristics but with no nonanginal symptoms or signs.

Heart associations which are trying to inform the public on how to recognize angina should probably not teach how to recognize typical angina, because then atypical angina, which may be more common than typical angina, will go undiagnosed. We also cannot teach the lay public that any chest pain could be atypical angina for fear of overloading our medical facilities with frightened patients who have nonanginal chest pains. One solution is to teach the public only how to diagnose nonanginal chest pain, and to suspect all pains that cannot be classified as nonanginal as possible angina.

\section{References}

1. Dwyer EM Jr, Weiner L, Cox JW: Angina pectoris in patients with normal and abnormal coronary arteriograms. Am J Cardiol 23: 639-646, 1969

2. Forsberg SA, Paulin S, Varnauskas E, Werko L: Coronary angiography in the diagnosis of coronary heart disease. Acta Med Scand 173: 269-279, 1963

3. Pasternak RC, Thibault GE, Savoia M, DeSanctis RW, Hutter AM: Chest pain with angiographically insignificant coronary arterial obstruction: Clinical presentation and long-term follow-up. Am J Med 68: 813-817, 1980

4. Lim HF, Dreifus SL, Kasparian H, Jajmi M, Balis G: Chest pain, coronary artery disease, and coronary cinearteriography. Chest 57: $41-46,1970$

.5 McElroy JB: Angina pectoris with coexisting skeletal chest pain. Am Heart J 66: 296-300, 1973

6. Calabro JJ, Jeghers H, Miller KA, Gordon RD: Classification of anterior chest wall syndromes. JAMA 243: 1420-1421, 1980

7. Epstein SE, Gerber LH: Chest wall syndrome. JAMA 241: 2793-2897, 1979

8. Wolf E, Stern S: Costosternal syndrome: Its frequency and importance in differential diagnosis of coronary heart disease. Arch Intern Med 136: 189-191, 1976

9. Sandler G, Illahi MA, Lawson CW: Glyceryl trinitrate in angina 
pectoris. Lancet 1: 1130-1136, 1963

10. Shimomura K, Murao S, Mashima S, Matsuo H, Murayama M, Yoshimoto N, Takayanagi K, Tseng YZ: Electrocardiographic findings of nocturnal angina: Comparison between angina with ST segment elevation and angina with ST segment depression. The 8th World Congress of Cardiology Abstract, Tokyo, 1978, 175

11. Merrill AJ Jr: Chest pain - the diagnostic dilemma. J Med Assoc Ga 65: 395-398, 1976

12. Buda A, Levene DL: The influence of inspiration on angina pectoris: a clue to right coronary artery disease. Am Heart J 92: 537, 1976

13. Neill WA, Hattenhauer M: Impairment of myocardial $\mathrm{O}_{2}$ supply due to hyperventilation. Circulation 52: $854-858,1975$

14. Astrand PO: Quantification of exercise capability. Prog Cardiovasc Dis 19: 51-61, 1976

15. Rinzler SH, Travell $\mathbf{J}$ : Therapy directed at the somatic component of cardiac pain. Am Heart J 35: 248-268, 1948

16. Burch GE, Giles TD: Cardiac causalgia. Arch Intern Med 125: $809-814,1970$

17. Alcalay M, Bontous D: Les syndromes douloureux de la paroi thoracique apres infarction due myocarde et apres chirgie cardiovasculaire. Coeur Med Inter. 13: 99-106, 1974

18. Henry JA, Montuschi E: Cardiac pain referred to site of previously experienced somatic pain. Br Med J 2: 1605-1606, 1978

19. Bennett JR, Atkinson M: The differentiation between esophageal and cardiac pain. Lancet 2: 1123-1127, 1966
20. Roberts R, Henderson RD, Wigle ED: Esophageal disease as a cause of severe retrosternal chest pain. Chest 67: 523-526, 1975

21. Kelly ML Jr: Atypical chest pain. Hosp Prac 13: 158-162, 1978

22. Frank PI: Some historical aspects of chest pain. Practitioner 211: 96-104, 1973

23. Levene DL: Some helpful hints. In: Chest Pain: An Integrated Diagnostic Approach, Levene DL, ed., Lea \& Febiger, Philadelphia 1977, 183-185

24. Wood P: DaCosta syndrome. Br Med J 1: 767-772, 1941

25. Horwitz LD: The diagnostic significance of anginal symptoms. JAMA 229: 1196-1199, 1974

26. King BM: Precordial catch. Lancet 2: 1035, 1959

27. Good AE: The chest pain of ankylosing spondylitis: its place in the differential diagnosis of heart pain. Ann Intern Med 58: 926937, 1963

28. Henderson RD, Wigle ED, Sample K, Marryatt G: Atypical chest pain of cardiac and esophageal origin. Chest 73: 24-27, 1978

29. Cohen BR: Evaluation of the patient with chest pain. Mod Treat 7: $1169-1181,1970$

30. Kohn RM, Cutcher B: Breath-holding time in the screening for rehabilitation potential of cardiac patients. Scand J Rehabil Med 2: $105-107,1970$

31. Sklaroff $\mathbf{H J}$ : Xiphodynia - another cause of atypical chest pain: six case reports. Mt Sinai J Med 40: 546-548, 1979 